\title{
ON CANONICAL REPRESENTATIONS AND BEREZIN KERNELS
}

\author{
GERRIT VAN DIJK \\ Mathematical Institute, Leiden University \\ P.O Box 9512, 2300 RA Leiden, The Netherlands \\ E-mail:dijk@math.leidenuniv.nl
}

1. Introduction. These are introductory lectures on canonical representations. The lectures aim to be an advertisement for a new kind of representations. Harmonic analysis is mainly concerned with $L^{2}$ Fourier analysis, so with the decomposition of regular representations. Canonical representations are different, and are to be regarded as quantizations of regular representations. They were introduced by Berezin in or about 1975 ([2], [3], [4], [5]), as well as by Gelfand, Graev and Vershik, from a different point of view, ([50], [51]). Their study is now a hot topic, mainly because of the relation with the decomposition of the tensor product of a holomorphic and anti-holomorphic discrete series representation. We restrict because of the introductory nature to the group $S U(1,1)$. At the end of the lectures we give some literature (included in the references) for further reading.

2. Unit disc and upper half plane. Let $G=S U(1,1)$ be the group of complex $2 \times 2$-matrices of the form

$$
\left(\begin{array}{ll}
\alpha & \beta \\
\bar{\beta} & \bar{\alpha}
\end{array}\right)
$$

satisfying $|\alpha|^{2}-|\beta|^{2}=1$. It acts transitively on the unit $\operatorname{disc} D=\{\zeta \in \mathbb{C}:|\zeta|<1\}$ by

$$
g \cdot \zeta=\frac{\alpha \zeta+\beta}{\bar{\beta} \zeta+\bar{\alpha}} .
$$

The stabilizer of $\zeta=0$ is the subgroup

$$
K=\left\{\left(\begin{array}{cl}
e^{i \theta} & 0 \\
0 & e^{-i \theta}
\end{array}\right): \theta \in \mathbb{R}\right\},
$$

2000 Mathematics Subject Classification: Primary 22E30.

Received 19 February 2001; revised 6 August 2001.

The paper is in final form and no version of it will be published elsewhere. 
so $D \simeq G / K$. We also consider the group $G_{o}=S L(2, \mathbb{R})$, acting on the Poincaré upper half plane $\mathbb{C}^{+}=\{z=x+i y: y>0\}$ by

$$
g \cdot z=\frac{a z+b}{c z+d}
$$

if $g=\left(\begin{array}{ll}a & b \\ c & d\end{array}\right)$. The stabilizer of $z=i$ is given by

$$
K_{o}=\left\{\left(\begin{array}{rr}
\cos \theta & \sin \theta \\
-\sin \theta & \cos \theta
\end{array}\right): \theta \in \mathbb{R}\right\},
$$

and hence $\mathbb{C}^{+} \simeq G_{o} / K_{o}$.

The Cayley transform $c: z \rightarrow \frac{z-i}{z+i}$ transforms $\mathbb{C}^{+}$bijectively onto $D$. Let $C$ be the $2 \times 2$ matrix

$$
\left(\begin{array}{rr}
1 & -i \\
1 & i
\end{array}\right)
$$

Then $G=C G_{o} C^{-1}$, and also, as transformation groups, $G=c G_{0} c^{-1}$. We call $D$ a bounded realization of $G / K$ and $\mathbb{C}^{+}$a tube realization of $G / K$.

3. Some subgroups of $G$ and $G_{o}$. Set

$$
A_{o}=\left\{a_{t}=\left(\begin{array}{cc}
\mathrm{e}^{t} & 0 \\
0 & \mathrm{e}^{-t}
\end{array}\right): t \in \mathbb{R}\right\}, \quad N_{o}=\left\{n_{\xi}=\left(\begin{array}{ll}
1 & \xi \\
0 & 1
\end{array}\right): \xi \in \mathbb{R}\right\} .
$$

These are subgroups of $G_{o}$ and $G_{o}=K_{o} A_{o} N_{o}=A_{o} N_{o} K_{o}$ (Iwasawa decomposition). The corresponding subgroups of $G$ are:

$$
A=\left\{\left(\begin{array}{cc}
\cosh t & \sinh t \\
\sinh t & \cosh t
\end{array}\right): t \in \mathbb{R}\right\} \text { and } N=\left\{\left(\begin{array}{cc}
1+\frac{i \xi}{2} & -\frac{i \xi}{2} \\
\frac{i \xi}{2} & 1-\frac{i \xi}{2}
\end{array}\right): \xi \in \mathbb{R}\right\} .
$$

4. The invariant measures and the Laplace-Beltrami operators on $D$ and $\mathbf{C}^{+} . D \simeq G / K$ and $\mathbb{C}^{+} \sim G_{o} / K_{o}$ carry invariant measures. Let us start with $\mathbb{C}^{+}$. Taking $d g=d t d \xi d k_{o}$ as Haar measure on $G_{o}\left(g=a_{t} n_{\xi} k\right), d k_{o}$ being the normalized Haar measure on $K_{o}$, we get for the $G_{o}$-invariant measure $d \mu$ on $\mathbb{C}^{+}$:

$$
d \mu(z)=\frac{1}{2} \frac{d x d y}{y^{2}}
$$

if $z=x+i y$. Similarly on $D$ :

$$
d \nu(\zeta)=2\left(1-|\zeta|^{2}\right)^{-2} d u d v
$$

where $\zeta=u+i v$, is the corresponding $G$-invariant measure.

The Laplace-Beltrami operator on $\mathbb{C}^{+}$is given by $y^{2}\left(\frac{\partial^{2}}{\partial x^{2}}+\frac{\partial^{2}}{\partial y^{2}}\right)$, and accordingly on $\mathcal{D}$ by the formula $\left(1-|\zeta|^{2}\right)^{2} \frac{\partial^{2}}{\partial \zeta \partial \bar{\zeta}}=4 v^{2}\left(\frac{\partial^{2}}{\partial u^{2}}+\frac{\partial^{2}}{\partial v^{2}}\right)$.

5. Berezin kernel, canonical representation. Set for $\lambda \in \mathbb{R}, g \in G$,

$$
\psi_{\lambda}(g)=\left(1-|\zeta|^{2}\right)^{\lambda}
$$

if $\zeta=g \cdot 0, \zeta \in D$. 
Clearly $\psi_{\lambda}$ is a bi- $K$-invariant continuous function on $G$. An easy computation shows:

$$
\psi_{\lambda}\left(g_{1}^{-1} g_{2}\right)=\left\{\frac{\left(1-|\zeta|^{2}\right)\left(1-|\eta|^{2}\right)}{(1-\zeta \bar{\eta})(1-\eta \bar{\zeta})}\right\}^{\lambda}
$$

if $\zeta=g_{1} \cdot 0, \eta=g_{2} \cdot 0$. Let us denote this expression by $B_{\lambda}(\eta, \zeta)$. $B_{\lambda}$ is called a Berezin kernel on $D$. Clearly $B_{\lambda}$ is $G$-invariant by construction: $B_{\lambda}(g \cdot \eta, g \cdot \zeta)=B_{\lambda}(\eta, \zeta)$ for all $g \in G$.

A kernel $\mathcal{K}(\eta, \zeta)$ on $\mathcal{D} \times \mathcal{D}$ is said to be positive-definite if

$$
\sum_{i=1}^{N} \mathcal{K}\left(\eta_{i}, \eta_{j}\right) \lambda_{i} \bar{\lambda}_{j} \geq 0
$$

for all finite sets of elements $\eta_{1}, \ldots, \eta_{N}$ in $\mathcal{D}$ and complex numbers $\lambda_{1}, \ldots, \lambda_{N}$. If $\mathcal{K}$ is continuous, this property is well-known (and easily shown by approximating with Dirac measures) to be equivalent to

$$
\int_{\mathcal{D}} \int_{\mathcal{D}} \mathcal{K}(\eta, \zeta) f(\zeta) \overline{f(\eta)} d \nu(\zeta) d \nu(\eta) \geq 0
$$

for all functions $f \in C_{c}^{\infty}(\mathcal{D})$, the space of $C^{\infty}$-functions on $\mathcal{D}$ with compact support.

Since products and (uniform) limits of positive-definite kernels are again positivedefinite, we easily get , by expanding $(1-\zeta \bar{\eta})^{-\lambda}$ into a power series:

$$
(1-\zeta \bar{\eta})^{-\lambda}=\sum_{m=0}^{\infty}\left(\begin{array}{c}
-\lambda \\
m
\end{array}\right) \zeta^{m} \bar{\eta}^{m}(-1)^{m}
$$

that $B_{\lambda}$ is a positive-definite kernel for $\lambda \geq 0$. Or, otherwise said, $\psi_{\lambda}$ is a positive-definite function for $\lambda \geq 0$ :

$$
\int_{G} \int_{G} \psi_{\lambda}\left(g_{2}^{-1} g_{1}\right) f\left(g_{1}\right) \overline{f\left(g_{2}\right)} d g_{1} d g_{2} \geq 0
$$

for all $f \in C_{c}^{\infty}(G / K)$, the space of $C_{c}^{\infty}$-functions on $G$, which are right-invariant with respect to the subgroup $K$.

Let $\tau_{\lambda}$ denote the unitary representation, naturally associated with $\psi_{\lambda}$ or $B_{\lambda}$. Its definition is straightforward. The Hilbert space is the completion of the pre-Hilbert space of functions of the form $f * \psi_{\lambda}$ with $f \in C_{c}^{\infty}(G / K)$ and inner product

$$
\left\langle f_{1} * \psi_{\lambda}, f_{2} * \psi_{\lambda}\right\rangle=\int_{G} \int_{G} \psi_{\lambda}\left(g_{2}^{-1} g_{1}\right) f_{1}\left(g_{1}\right) \overline{f\left(g_{2}\right)} d g_{1} d g_{2}
$$

This is a well-defined expression, which produces a genuine inner product. The right-hand side can also be written as

$$
\int_{G} \psi_{\lambda}(g)\left(\widetilde{f_{2}} * f_{1}\right)(g) d g
$$

where $\widetilde{f}_{2}(x)=\overline{f_{2}\left(x^{-1}\right)}, x \in G$. The $G$-action is simply left translation.

We call the $\tau_{\lambda}(\lambda \geq 0)$ canonical representations after Gelfand, Graev and Vershik (1975), cf. [50], [51]. We shall study these representations; in particular their behaviour for $\lambda \rightarrow \infty$ and their decomposition into irreducible representations. 
6. The spherical Fourier transform of $\psi_{\lambda}$. For $s \in \mathbb{C}$ let

$$
\varphi_{s}(g)=\int_{K} \mathrm{e}^{(-s+1) t(k g)} d k \quad(g \in G)
$$

where $t(g)$ is given by the Iwasawa decomposition $g=a_{t(g)} n k$. Then $\varphi_{s}$ is a zonal spherical function with the parametrization of Harish-Chandra.

We also need Harish-Chandra's $c$-function:

$$
c(s)=2^{1-s} \frac{\Gamma(s)}{\Gamma\left(\frac{s+1}{2}\right)^{2}} .
$$

Let $\psi_{\lambda}$ be as in Section 5 . It easily follows from the explicit form of the invariant measure on $D$ that $\psi_{\lambda} \in L^{1}(G) \cap L^{2}(G)$ for $\lambda>1$ (even for $\operatorname{Re} \lambda>1$, though $\psi_{\lambda}$ was a priori defined for real $\lambda$ only).

To decompose the canonical representation $\tau_{\lambda}$ into irreducible unitary representations for $\lambda>1$, it suffices to determine the inverse spherical Fourier transform of $\psi_{\lambda}$ :

$$
\psi_{\lambda}(g)=\frac{1}{\pi^{2}} \int_{0}^{\infty} a_{\lambda}(i \mu) \varphi_{i \mu}(g) \frac{d \mu}{|c(i \mu)|^{2}}
$$

in $L^{2}$-sense, where $a_{\lambda}(i \mu)$ is the spherical Fourier transform of $\psi_{\lambda}$ :

$$
a_{\lambda}(i \mu)=\int_{G} \psi_{\lambda}(g) \varphi_{-i \mu}(g) d g .
$$

It will turn out that $a_{\lambda}(i \mu)$ is also of importance for $0<\lambda \leq 1$.

7. Computation of $a_{\lambda}(i \mu)$. Notice that

$$
a_{\lambda}(i \mu)=\int_{G} \psi_{\lambda}(g) \mathrm{e}^{(+i \mu+1) t(g)} d g
$$

and that this is actually an integral over $G / K$. This integral is most easily computed in the tube realization of $G / K$, namely on $\mathbb{C}^{+}$.

Then

$$
\psi_{\lambda}(z)=\left(1-|c z|^{2}\right)^{\lambda}=\left(\frac{4 y}{x^{2}+(y+1)^{2}}\right)^{\lambda}
$$

if $z=x+i y, y>0$. Here $c$ is the Cayley transform.

Set $\Delta(z)=y$. Then $\Delta\left(n_{\xi} \cdot z\right)=\Delta(z)$ and $\Delta\left(a_{t} \cdot z\right)=\mathrm{e}^{2 t} \Delta(z)$. Therefore

$$
\begin{aligned}
a_{\lambda}(i \mu) & =\int_{\mathbb{C}^{+}} \psi_{\lambda}(z) \Delta(z)^{\frac{1}{2} i \mu+\frac{1}{2}} d \mu(z) \\
& =\pi \frac{\Gamma\left(\lambda+\frac{i \mu-1}{2}\right) \Gamma\left(\lambda+\frac{-i \mu-1}{2}\right)}{\Gamma(\lambda)^{2}} .
\end{aligned}
$$

For a computation, applying the Cartan decomposition $G=K A K$, see [10]. This computation relies on formulae in [20].

8. Behaviour as $\lambda \rightarrow \infty$. The following is well known (see [20] p. 47, (1)):

$$
\frac{\Gamma(z+a)}{\Gamma(z+b)}=z^{a-b}\left\{1+\frac{1}{2 z}(a-b)(a+b-1)+\mathcal{O}\left(z^{-2}\right)\right\} \text { as } z \rightarrow \infty \text {. }
$$


This leads to:

$$
\frac{\lambda-1}{\pi} a_{\lambda}(i \mu) \sim 1+\frac{-\mu^{2}-1}{4 \lambda}+\cdots(\lambda \rightarrow \infty) .
$$

So, up to a right normalization, $\tau_{\lambda}$ tends to the usual regular representation of $G$ on $G / K \simeq D$. The normalization factor is $\frac{\lambda-1}{\pi}$.

Indeed, we also have, because $\square \varphi_{s}=\left(s^{2}-1\right) \varphi_{s}$,

$$
\frac{\lambda-1}{\pi} \psi_{\lambda}=\delta+\frac{\square \delta}{4 \lambda}+\mathcal{O}\left(\frac{1}{\lambda^{2}}\right),
$$

where $\square$ is the Laplace-Beltrami operator, and thus obtain for all $f_{1}, f_{2} \in C_{c}^{\infty}(G / K)$ that

$$
\frac{\lambda-1}{\pi} \int_{G} \psi_{\lambda}(g)\left(\widetilde{f}_{2} * f_{1}\right)(g) d g
$$

tends to

$$
\int_{G} \delta(g)\left(\widetilde { f } * f _ { 1 } \left((g) d g=\int_{G} f_{1}(g) \overline{f_{2}(g)} d g,\right.\right.
$$

which is the usual $L^{2}$-inner product of $f_{1}$ and $f_{2}$ on $G / K$.

9. Decomposition of $\tau_{\lambda}$. Set

$$
a_{\lambda}(s)=\pi \frac{\Gamma\left(\lambda+\frac{s-1}{2}\right) \Gamma\left(\lambda+\frac{-s-1}{2}\right)}{\Gamma(\lambda)^{2}} .
$$

Then we can write for $\operatorname{Re} \lambda>1$ :

$$
\psi_{\lambda}=\frac{1}{2 i \pi^{2}} \int_{C} a_{\lambda}(s) \varphi_{s} \frac{d s}{c(s) c(-s)},
$$

where $C$ is the line $\operatorname{Re} s=0$ in the complex plane, taken from below upwards. The integrand in (9.1) has, as a function of $s$, poles depending on $\lambda$ at $s= \pm(1-2 \lambda-2 k), k=$ $0,1,2, \ldots$. So $(9.1)$ even holds for $\operatorname{Re} \lambda>1 / 2$.

For $\operatorname{Re} \lambda>1 / 2$ we may shift $C$ to $C^{\prime}$ (see figure) and the resulting integral is analytic in $\lambda$ for $\operatorname{Re} \lambda \rightarrow 0$. When $\operatorname{Re} \lambda \rightarrow 0, \pm(1-2 \lambda)$ pass $C$.

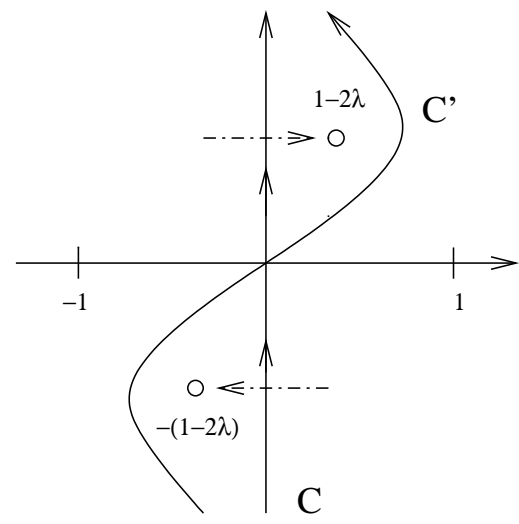

When shifting $C^{\prime}$ to $C$ again, we meet the poles $\pm(1-2 \lambda)$, but only for $0<\operatorname{Re} \lambda<1 / 2$. 
(The case $\operatorname{Re} \lambda=1 / 2$ causes trouble). The residue of $\frac{a_{\lambda}(s)}{c(s) c(-s)}$ at $1-2 \lambda$ is equal to

$$
r(\lambda)=\frac{\pi}{2} \frac{\Gamma(1-\lambda)^{2}}{\Gamma(1-2 \lambda)} .
$$

So we get for $0<\operatorname{Re} \lambda<1 / 2$ :

$$
\psi_{\lambda}=\frac{1}{\pi^{2}} \int_{0}^{\infty} a_{\lambda}(i \mu) \varphi_{i \mu} \frac{d \mu}{|c(i \mu)|^{2}}+\frac{\Gamma(1-\lambda)^{2}}{\Gamma(1-2 \lambda)} \varphi_{1-2 \lambda}
$$

in the sense of distributions.

Let us now consider the case $\lambda=1 / 2$ (only). Then we would like to take the limit in (9.1) from the right and in (9.2) from the left. This is allowed and gives no residue. Of course, the integrand $\frac{a_{\lambda}(s)}{c(s) c(-s)}$ at first sight has a singularity at $s=0 ; a_{\lambda}(s)$ has a double pole, $\frac{1}{c(s) c(-s)}$ a double zero, so actually everything is in order.

The spherical function $\varphi_{1-2 \lambda}$ is positive-definite and corresponds to the complementary series of $G$, see next section, for $\lambda \in \mathbb{R}, 0<\lambda<1 / 2$.

Observe that (9.2) also holds for $\lambda=0$.

A similar calculation as the above gives the analytic continuation beyond $\lambda=0$, namely for $\lambda<0$. If

$$
-\frac{1}{2}-k<\operatorname{Re} \lambda<\frac{1}{2}-k, k=0,1,2, \ldots
$$

then we get $k+1$ extra terms $R_{j}(\lambda) \varphi_{1-2 \lambda-2 j}$ with

$$
R_{j}(\lambda)=\frac{\Gamma(1-\lambda)^{2} \cdot(1-2 \lambda-2 j)}{j ! \Gamma(2-2 \lambda-j)}
$$

$(j=0, \ldots, k)$. In other words: if $-\frac{1}{2}-k<\operatorname{Re} \lambda<\frac{1}{2}-k$, then

$$
\psi_{\lambda}=\frac{1}{\pi^{2}} \int_{0}^{\infty} a_{\lambda}(i \mu) \varphi_{i \mu} \frac{d \mu}{|c(i \mu)|^{2}}+\sum_{j=0}^{k} R_{j}(\lambda) \varphi_{1-2 \lambda-2 j} .
$$

Of course unitarity of $\tau_{\lambda}$ fails for negative $\lambda$. It would be interesting to give a meaning to the limit for $\lambda$ integer and $\lambda \rightarrow-\infty$. Observe that the integral in (9.3) disappears for $\lambda$ a negative integer. It is plausible that the (normalized) limit gives the Plancherel formula for $\mathrm{SU}(2) / K$. Might we say that the $\tau_{\lambda}$ yield an interpolation between $L^{2}(\mathrm{SU}(2) / K)$ and $L^{2}(\mathrm{SU}(1,1) / K) ?^{1}$

10. Complementary series representations; a model. Let $U$ denote the unit circle in $\mathbb{C}$, the boundary of $D$. $G$ acts on $U$ by fractional linear transformations:

$$
g \cdot \omega=\frac{\alpha \omega+\beta}{\bar{\beta} \omega+\bar{\alpha}}
$$

\footnotetext{
${ }^{1}$ This question is very recently settled by Yu. A. Neretin in his preprint: Matrix balls, radial analysis of Berezin kernels, and hypergeometric determinants, http://xxx.lanl.gov/ math.RT/0012200. A careful analysis of the asymptotic behaviour for $\lambda \rightarrow-\infty$ shows that even the "correspondence principle", i.e. the analogue of formula (8.2) holds. See a forthcoming note by van Dijk and Molchanov. We will return to this matter in Section 14.
} 
if $g=\left(\frac{\alpha}{\beta} \frac{\beta}{\alpha}\right), \omega \in U$.

For $s \in \mathbb{C}$ we define a representation $\pi_{s}^{c}$ of $G$ acting on $C(U)$ :

$$
\pi_{s}^{c}(g) f(\omega)=|\bar{\beta} \omega+\bar{\alpha}|^{-(s+1)} f\left(g^{-1} \cdot \omega\right)
$$

if $g^{-1}=\left(\frac{\alpha}{\beta} \frac{\beta}{\alpha}\right)$.

We provide $C(U)$ with the sesquilinear form

$$
(f, g)_{s}=\int_{U} \int_{U}\left|1-\omega \bar{\omega}^{\prime}\right|^{s-1} f(\omega) \overline{g\left(\omega^{\prime}\right)} d \omega d \omega^{\prime} \quad(s>0)
$$

where $d \omega$ is $\frac{1}{2 \pi} d \theta$ if $\omega=\mathrm{e}^{i \theta}$. Then $d \omega$ is quasi-invariant with respect to the action of $G$ on $U$ :

$$
\int_{U} f(g \cdot \omega) d \omega=\int_{U} f(\omega)|\bar{\beta} \omega+\bar{\alpha}|^{-2} d \omega
$$

Thus, $(,)_{s}$ is $G$-invariant with respect to $\pi_{s}^{c}$. It turns out that $(,)_{s}$ is positive-definite for $0<s<1$. The corresponding series of (irreducible) unitary representations is called the complementary series of $G$.

REMARK. With respect to the usual $L^{2}$-inner product on $C(U)$, we get the spherical principal series $\pi_{s}$. For $s \in i \mathbb{R}, \pi_{s}$ is unitary and irreducible.

\section{The holomorphic and anti-holomorphic discrete series; canonical repre-} sentations as tensor products. For $\lambda \in \mathbb{R}$ consider the Fock space $\mathcal{F}_{\lambda}$ of holomorphic functions on $D$ satisfying

$$
\|f\|_{\lambda}^{2}=\int_{D}|f(\zeta)|^{2}\left(1-|\zeta|^{2}\right)^{\lambda} d \nu(\zeta)<\infty
$$

This space is non-trivial for $\lambda>1$, since $\mathcal{F}_{\lambda}$ contains the function which is identically 1 . One has

$$
\|1\|_{\lambda}^{2}=\frac{\pi}{\lambda-1}
$$

$\mathcal{F}_{\lambda}$ is a closed subspace of $L^{2}\left(D, d \nu_{\lambda}\right)$, hence a Hilbert space, where $d \nu_{\lambda}(\zeta)=(1-$ $\left.|\zeta|^{2}\right)^{\lambda} d \nu(\zeta)$. It has a reproducing kernel, namely

$$
E_{\lambda}(\zeta, \eta)=\frac{\lambda-1}{\pi}(1-\zeta \bar{\eta})^{-\lambda} .
$$

It is also a unitary module for the action of the universal covering group $\widetilde{G}$ of $G$; for integer $\lambda>1$ it is even a $G$-module: a holomorphic discrete series representation. The group $G$ acts by

$$
\pi_{\lambda}^{d}(g) f(\zeta)=f\left(\frac{\alpha \zeta+\beta}{\bar{\beta} \zeta+\bar{\alpha}}\right)(\bar{\beta} \zeta+\bar{\alpha})^{-\lambda}
$$

if $g^{-1}=\left(\frac{\alpha}{\beta} \frac{\beta}{\alpha}\right)$.

Let us denote by $\overline{\mathcal{F}}_{\lambda}$ the space of complex conjugates of elements of $\mathcal{F}_{\lambda}$. It consists of anti-holomorphic functions and gives rise to an obvious unitary action $\bar{\pi}_{\lambda}^{d}$ of $\widetilde{G}$ as well. So

$$
\bar{\pi}_{\lambda}^{d}(g) f(\zeta)=f\left(\frac{\alpha \zeta+\beta}{\bar{\beta} \zeta+\bar{\alpha}}\right)(\beta \bar{\zeta}+\alpha)^{-\lambda}
$$


if $g^{-1}=\left(\frac{\alpha}{\beta} \frac{\beta}{\alpha}\right), f \in \overline{\mathcal{F}}_{\lambda}, \lambda>1$. For $\lambda \in \mathbb{N}, \lambda>1$ we get the anti-holomorphic discrete series of $G$.

Consider the Hilbert space tensor product $\mathcal{F}_{\lambda} \widehat{\otimes}_{2} \overline{\mathcal{F}}_{\lambda}$ with $\lambda>1$. The group $\widetilde{G}$ acts diagonally. It turns out that we actually have a $G$-action, which for integer $\lambda$ is given by

$$
g_{o} \cdot F(\zeta, \eta)=F\left(g_{o}^{-1} \cdot \zeta, g_{o}^{-1} \cdot \eta\right)(\bar{\alpha}+\bar{\beta} \zeta)^{-\lambda}(\alpha+\beta \bar{\eta})^{-\lambda}
$$

if $g_{o}^{-1}=\left(\frac{\alpha}{\beta} \frac{\beta}{\alpha}\right)$.

Let $A_{\lambda}$ denote the linear map, defined on $\mathcal{F}_{\lambda} \widehat{\otimes}_{2} \overline{\mathcal{F}}_{\lambda}$ by

$$
F(\zeta, \eta) \rightarrow F(\zeta, \zeta)\left(1-|\zeta|^{2}\right)^{\lambda}
$$

$A_{\lambda}$ is a bounded linear operator $\mathcal{F}_{\lambda} \widehat{\otimes}_{2} \overline{\mathcal{F}}_{\lambda} \rightarrow L^{2}(D, d \nu)$ where $L^{2}(D, d \nu)$ is considered as a $G$-space in the natural way. $A_{\lambda}$ intertwines the $G$-actions. Let us show that $A_{\lambda}$ is bounded.

Let $F(\zeta, \eta) \in \mathcal{F}_{\lambda} \widehat{\otimes}_{2} \overline{\mathcal{F}}_{\lambda}$. Then, according to the reproducing property,

$$
F(\zeta, \eta)=\int_{D} E_{\lambda}(w, \eta) F(\zeta, w) d \nu_{\lambda}(w)
$$

therefore

$$
A_{\lambda} F(\zeta)=\int_{D} E_{\lambda}(w, \zeta) F(\zeta, w) d \nu_{\lambda}(w) \cdot\left(1-|\zeta|^{2}\right)^{\lambda}
$$

Hence

$$
\left|A_{\lambda} F(\zeta)\right|^{2} \leq \int_{D}\left|E_{\lambda}(w, \zeta)\right|^{2} d \nu_{\lambda}(w) \cdot \int_{D}|F(\zeta, w)|^{2} d \nu_{\lambda}(w) \cdot\left(1-|\zeta|^{2}\right)^{2 \lambda}
$$

So

$$
\left\|A_{\lambda} F\right\|^{2}=\int_{D}\left|A_{\lambda} F(\zeta)\right|^{2} d \nu(\zeta) \leq \frac{1}{\|1\|_{\lambda}^{2}} \cdot\|F\|_{2}^{2} .
$$

We now determine $A_{\lambda}^{*}$.

Let $F(\zeta, \eta)$ be holomorphic in $\zeta$, anti-holomorphic in $\eta$, and belonging to $\mathcal{F}_{\lambda} \widehat{\otimes}_{2} \overline{\mathcal{F}}_{\lambda}$. Let $h \in L^{2}(D, d \nu)$. We shall determine an explicit expression for $A_{\lambda}^{*} h$. It is clear that $A_{\lambda}^{*} h(\zeta, \eta)$ is holomorphic in $\zeta$ and anti-holomorphic in $\eta$, since it belongs to $\mathcal{F}_{\lambda} \widehat{\otimes}_{2} \overline{\mathcal{F}}_{\lambda}$.

One has:

$$
\begin{aligned}
\left(A_{\lambda}^{*} h, F\right) & =\left(h, A_{\lambda} F\right) \\
& =\int_{D} \int_{D} h(z) E_{\lambda}(z, w) \bar{F}(z, w)\left(1-|z|^{2}\right)^{\lambda} d \nu_{\lambda}(w) d \nu(z) .
\end{aligned}
$$

So $A_{\lambda}^{*} h$ is the projection of the function

$$
(z, w) \rightarrow h(z) E_{\lambda}(z, w)
$$

onto $\mathcal{F}_{\lambda} \widehat{\otimes}_{2} \overline{\mathcal{F}}_{\lambda}$. This function is in $L^{2}\left(D \times D, d \nu_{\lambda} \otimes d \nu_{\lambda}\right)$. The orthogonal projection, call it $E$, is given by

$$
E F(z, w)=\int_{D} \int_{D} E_{\lambda}\left(w^{\prime}, w\right) E_{\lambda}\left(z, z^{\prime}\right) F\left(z^{\prime}, w^{\prime}\right) d \nu_{\lambda}\left(z^{\prime}\right) d \nu_{\lambda}\left(w^{\prime}\right) .
$$

Hence

$$
A_{\lambda}^{*} h(\zeta, \eta)=\int_{D} E_{\lambda}\left(\zeta, z^{\prime}\right) E_{\lambda}\left(z^{\prime}, \eta\right) h\left(z^{\prime}\right) d \nu_{\lambda}\left(z^{\prime}\right)
$$


Finally,

$$
A_{\lambda} A_{\lambda}^{*} f(\zeta)=\int_{D} E_{\lambda}(\zeta, \eta) E_{\lambda}(\eta, \zeta) f(\eta) d \nu_{\lambda}(\eta) \cdot\left(1-|\zeta|^{2}\right)^{\lambda}
$$

So $A_{\lambda} A_{\lambda}^{*}$ is a kernel operator on $L^{2}(D, d \nu)$ with kernel:

$$
\begin{aligned}
B_{\lambda}(\zeta, \eta) & =\left(\|1\|_{\lambda}^{2}\right)^{-2} \frac{E_{\lambda}(\zeta, \eta) E_{\lambda}(\eta, \zeta)}{E_{\lambda}(\zeta, \zeta) E_{\lambda}(\eta, \eta)} \\
& =\left(\|1\|_{\lambda}^{2}\right)^{-2}\left\{\frac{\left(1-|\zeta|^{2}\right)\left(1-|\eta|^{2}\right)}{(1-\zeta \bar{\eta})(1-\bar{\zeta} \eta)}\right\}^{\lambda} .
\end{aligned}
$$

This is again the Berezin kernel up to a positive constant.

Consequently, applying the well-known theory of L. Schwartz on invariant Hilbert subspaces (cf. [7]), we get $\tau_{\lambda} \simeq$ restriction of $\pi_{\lambda}^{d} \widehat{\otimes}_{2} \bar{\pi}_{\lambda}^{d}$ to $G$.

12. Principal series of $S L(2, \mathbf{C})$; connection with canonical representations. Let $G_{c}=S L(2, \mathbb{C})$, a complexification of $G=S U(1,1)$. Let $P_{c}^{-}$be the Borel subgroup of $G_{c}$ consisting of the lower triangular matrices

$$
P_{c}^{-}:\left(\begin{array}{ll}
a & 0 \\
c & a^{-1}
\end{array}\right)
$$

with $c \in \mathbb{C}, a \in \mathbb{C}^{*}$ and let $P_{c}^{+}$be the group of upper triangular matrices

$$
P_{c}^{-}:\left(\begin{array}{ll}
a & b \\
0 & a^{-1}
\end{array}\right)
$$

with $b \in \mathbb{C}, a \in \mathbb{C}^{*} . G_{c}$ acts on $S=\left\{s \in \mathbb{C}^{2}:\|s\|^{2}=1\right\}$ and on $\widetilde{S}=S / \sim$, where $s \sim s^{\prime}$ iff $s=\lambda s^{\prime},|\lambda|=1$, by

$$
g \cdot s=\frac{g(s)}{\|g(s)\|}
$$

transitively. Clearly Stab $(\widetilde{0,1})=P_{c}^{-}$. So, $\widetilde{S} \simeq G_{c} / P_{c}^{-}$. Similarly $\widetilde{S} \simeq G_{c} / P_{c}^{+}$. If $d s$ is the usual normalized surface measure on $S$, then

$$
d(g \cdot s)=\|g(s)\|^{-4} d s .
$$

For $\mu \in \mathbb{C}$, define the character $\omega_{\mu}$ of $P_{c}^{ \pm}$by

$$
\omega_{\mu}(p)=|a|^{\mu} .
$$

Consider $\pi_{\mu}^{ \pm}=\operatorname{Ind}_{P_{c}^{ \pm} \uparrow G_{c}} \omega_{\mp \mu}$.

$\pi_{\mu}^{+}$and $\pi_{\mu}^{-}$can both be realized on $C^{\infty}(\widetilde{S})$ : the space of $C^{\infty}$-functions $\varphi$ on $S$ satisfying $\varphi(\lambda s)=\varphi(s)$ for $s \in S, \lambda \in \mathbb{C},|\lambda|=1$.

The formula for $\pi_{\mu}^{-}$is:

$$
\pi_{\mu}^{-}(g) \varphi(s)=\varphi\left(g^{-1} \cdot s\right)\left\|g^{-1}(s)\right\|^{\mu} .
$$

Let $\theta$ be the Cartan involution of $G_{c}$ given by $\theta(g)=\left(g^{*}\right)^{-1}$. Then

$$
\pi_{\mu}^{+}(g) \varphi(s)=\varphi\left(\theta\left(g^{-1}\right) \cdot s\right) \| \theta\left(g^{-1}(s) \|^{\mu} .\right.
$$


Let $\left(\right.$, ) denote the standard inner product on $L^{2}(S)$ :

$$
(\varphi, \psi)=\int_{S} \varphi(s) \bar{\psi}(s) d s .
$$

Then clearly this form is invariant with respect to the pairs

$$
\left(\pi_{\mu}^{-}, \pi_{-\bar{\mu}-4}^{-}\right) \text {and }\left(\pi_{\mu}^{+}, \pi_{-\bar{\mu}-4}^{+}\right) .
$$

Therefore, if $\operatorname{Re} \mu=-2$, then the representations $\pi_{\mu}^{ \pm}$are unitarizable, the inner product being $($,$) .$

Irreducibility and intertwining operators. It is known that:

- $\pi_{\mu}^{ \pm}$is irreducible if $\mu \notin\left(-4-2 \mathbb{N}_{0}\right) \cup\left(2 \mathbb{N}_{0}\right)$;

- if $\mu=2 k(k=0,1,2, \ldots)$, then there is one irreducible finite-dimensional subspace for $\pi_{\mu}^{ \pm}$, namely $\oplus_{a=0}^{k} \mathcal{H}_{a}\left(\mathcal{H}_{a}\right.$ : space of harmonic polynomials on $\mathbb{C}^{2}$, homogeneous of degree $a$ in $z$ and $\bar{z}$ ). The quotient representation is irreducible.

- if $\mu=-2 k-4$, then there is one infinite-dimensional irreducible subspace for $\pi_{\mu}^{ \pm}$, namely $\oplus_{a=k+1}^{\infty} \mathcal{H}_{a}$. The quotient representation is irreducible and finite-dimensional.

- $\pi_{\mu}^{+} \sim \pi_{-\mu-4}^{-}$(if $\pi_{\mu}^{+}$is irreducible), $\pi_{\mu}^{+} \sim \pi_{\mu}^{-}$.

- $\pi_{\mu}^{ \pm}$is unitarizable if and only if

- $\operatorname{Re} \mu=-2: \quad$ (spherical) principal series

- $\mu \in(-2,0)$ : complementary series.

Define the operator $A_{\mu}$ on $C^{\infty}(\widetilde{S})$ by the formula

$$
A_{\mu} \varphi(s)=\int_{S}|(s, t)|^{-\mu-4} \varphi(t) d t .
$$

The integral is absolutely convergent for $\operatorname{Re} \mu<-3$ and can be analytically extended to the whole complex plane as a meromorphic function. It is easily checked that $A_{\mu}$ is an intertwining operator

$$
A_{\mu} \pi_{\mu}^{ \pm}(g)=\pi_{-\mu-4}^{\mp} A_{\mu} .
$$

The operator $A_{-\mu-4} \cdot A_{\mu}$ intertwines $\pi_{\mu}^{ \pm}$with itself, and is therefore a scalar $c(\mu)$. It can be computed using $K$-types:

$$
c(\mu)=-\left(\frac{\mu}{2}+1\right)^{2} .
$$

$G$ acts on $S(G=S U(1,1)$, seen as the group of $2 \times 2$ matrices, with determinant 1, leaving $\left|z_{1}\right|^{2}-\left|z_{2}\right|^{2}$ invariant; set $\left.[z, w]=z_{1} \bar{w}_{1}-z_{2} \bar{w}_{2}\left(z, w \in \mathbb{C}^{2}\right)\right)$.

There are 3 orbits, given by

$$
[s, s]>0, \quad[s, s]=0, \quad[s, s]<0 .
$$

All three orbits are invariant under $s \rightarrow \lambda s(|\lambda|=1)$. Call the corresponding orbits on $\widetilde{S}: \mathcal{O}_{1}, \mathcal{O}_{2}, \mathcal{O}_{3}$. Then

$$
\begin{gathered}
\mathcal{O}_{1} \simeq G / K \quad \text { via } \quad g \rightarrow g \cdot e_{1} \\
\mathcal{O}_{2} \simeq G / M A N \quad \text { via } \quad g \rightarrow g \cdot\left(e_{1}+e_{2}\right) \\
\mathcal{O}_{3} \simeq G / K \quad \text { via } \quad g \rightarrow g \cdot e_{2} .
\end{gathered}
$$


Here $M=\{ \pm I\}, M A N$ is a minimal parabolic subgroup of $G$ and $e_{i}$ is the $i$-th unit vector in $\mathbb{C}^{2}$.

Let $\varphi$ be a $C^{\infty}$-function on $\mathcal{O}_{1}$. Then

$$
\psi(s)=[s, s]^{-\mu / 2} \varphi(s)
$$

again lives on $\mathcal{O}_{1}$ and satisfies

$$
\begin{aligned}
\psi(g \cdot s) & =[g \cdot s, g \cdot s]^{-\mu / 2} \varphi(g \cdot s) \\
& =\pi_{\mu}^{-}(g) \varphi(s)[s, s]^{-\mu / 2} \quad(g \in G) .
\end{aligned}
$$

So $\varphi \rightarrow \psi$ is a linear intertwiner of $\pi_{\mu}^{-} \mid G$ with the left regular action of $G$ on $C_{c}^{\infty}(G / K)=$ $C_{c}^{\infty}\left(\mathcal{O}_{1}\right)=\mathcal{D}\left(\mathcal{O}_{1}\right)$. Since $\theta(g)=J g J(g \in G), J=\operatorname{diag}(1,-1), A_{\mu}$ becomes

$$
A_{\mu} \varphi(s)=\int_{S}|[s, t]|^{-\mu-4} \varphi(t) d t \quad\left(s \in \mathcal{O}_{1}\right)
$$

and is defined on $C_{c}^{\infty}\left(\mathcal{O}_{1}\right)$ for all $\mu$, and intertwines $\pi_{\mu}^{-}$and $\pi_{-\bar{\mu}-4}^{-}$restricted to $G$.

For $\varphi_{1}, \varphi_{2} \in C_{c}^{\infty}\left(\mathcal{O}_{1}\right)$ and $\mu \in \mathbb{R}$ consider the Hermitian form

$$
\left\langle\varphi_{1}, A_{\mu} \varphi_{2}\right\rangle=\int_{S} \int_{S}|[s, t]|^{-\mu-4} \varphi_{1}(s) \overline{\varphi_{2}(t)} d s d t .
$$

This form is clearly $\pi_{\mu}^{-}(G)$-invariant. A $G$-invariant measure on $\mathcal{O}_{1}$ is given by $d m(s)=$ $\frac{d s}{[s, s]^{2}}$. Applying the above linear map $\varphi \rightarrow \psi$ we get instead of (12.13),

$$
\left\langle\psi_{1}, \psi_{2}\right\rangle_{\mu}=\int_{S} \int_{S} \psi_{1}(s) \overline{\psi_{2}(t)}\left[\frac{[s, s][t, t]}{[s, t][t, s]}\right]^{\frac{\mu}{2}+2} d m(s) d m(t) .
$$

Thus

$$
B_{\lambda}(s, t)=\left\{\frac{[s, s][t, t]}{[s, t][t, s]}\right\}^{\lambda}
$$

is again the Berezin kernel, now living on $[s, s]>0(s \in \widetilde{S}) \simeq \mathcal{O}_{1}$. Indeed, $B_{\lambda}\left(g \cdot e_{1}, e_{1}\right)=$ $(\cosh t)^{-2 \lambda}$ if $g=a_{t}$.

This approach was firstly observed by Molchanov in [33].

\section{Some generalizations and remarks}

- Let us restrict the holomorphic discrete series $\pi_{\lambda}^{d}$ to the real group $S O_{o}(1,1)$ and the functions in $\mathcal{F}_{\lambda}$ to the real "unit disc" $(x \in \mathbb{R},|x|<1)$. More precisely, let us define the analogue of $A_{\lambda}$ by

$$
F(\zeta) \rightarrow F(x)\left(1-|x|^{2}\right)^{\lambda / 2} .
$$

Then we get "the" canonical representation of $S O_{0}(1,1)$ with Berezin kernel $B_{\lambda / 2}(x, y)$, which remains positive-definite for $\lambda>0$.

Let $\psi_{\lambda / 2}(x)=\left(1-|x|^{2}\right)^{\lambda / 2}$ be the associated positive-definite function. We can compute the spherical decomposition of this restricted representation in a similar way as before and get:

$$
a_{\lambda / 2}(i \mu)=2^{\lambda-1} \frac{\Gamma\left(\frac{\lambda+i \mu}{2}\right) \Gamma\left(\frac{\lambda-i \mu}{2}\right)}{\Gamma(\lambda)}
$$




$$
\begin{aligned}
c(s) & =\frac{1}{2} \\
\psi_{\lambda / 2}(g) & =\frac{1}{4 \pi} \int_{0}^{\infty} a_{\lambda / 2}(i \mu) \varphi_{i \mu}(g) \frac{d \mu}{|c(i \mu)|^{2}} \quad \text { in } L^{2} \text {-sense }
\end{aligned}
$$

If $g=a_{t}=\left(\begin{array}{l}\cosh t \sinh t \\ \sinh t \cosh t\end{array}\right)$, then $\varphi_{i \mu}(g)=\mathrm{e}^{-i \mu t}$. Observe that this decomposition holds for $\operatorname{Re} \lambda>0$.

The computation of $a_{\lambda / 2}(i \mu)$ is again most easily done in the tube realization. Observe that $|x|<1$ is identified with the positive imaginary axis. The integral becomes:

$$
2^{\lambda-1} \int_{0}^{\infty} \frac{y^{\lambda / 2+i \mu / 2}}{(y+1)^{\lambda}} \frac{d y}{y} .
$$

We thus finally get:

$$
(\cosh t)^{-\lambda}=\psi_{\lambda / 2}\left(a_{t}\right)=\frac{1}{2 \pi} \int_{0}^{\infty} \frac{2^{\lambda-1} \Gamma\left(\frac{\lambda+i \mu}{2}\right) \Gamma\left(\frac{\lambda-i \mu}{2}\right)}{\Gamma(\lambda)} \mathrm{e}^{-i \mu t} d \mu \quad(\operatorname{Re} \lambda>0)
$$

in $L^{2}$-sense, a "classical" Fourier transform identity. See [8] for generalization.

- A quite interesting extension of the notion of canonical representation is obtained by considering $\pi_{\lambda}^{d} \widehat{\otimes}_{2} \pi_{\lambda^{\prime}}^{d}$ with $\lambda-\lambda^{\prime}=l \in \mathbb{Z}$, see Section 11 (exercise). See [8], [52].

If $|l|>1$ we get discrete series occurring in the decomposition of " $\tau_{\lambda, l}$ " (apart from principal series and complementary series if $l$ is even).

- Extension of the results to $G=S U(1, n)$ is rather immediate. Finitely many complementary series occur for $\lambda>0$, situated at the points $n-2 \lambda-2 k=s_{k}$, as far as $s_{k}>0$. See $[10]$.

An interesting phenomenon occurs also in the "compact" realization of $\tau_{\lambda}$. The $G$ orbits on $\widetilde{S}=\left\{x \in \mathbb{C}^{n}:\|x\|<1\right\} / \sim$ can be identified with $G / K, G / P, G / H$, where $H=S(U(1, n-1) \times U(1))$. The Berezin kernel can also be defined on the latter space and gives rise to a canonical representation on a pseudo-Riemannian space, see [11]. Unfortunately, $\tau_{\lambda}$ is not unitary anymore, so a good decomposition theory is missing! One can however try to decompose $\psi_{\lambda}$ into $H$-spherical (positive-definite) distributions. (see Van Dijk-Molchanov, forthcoming publication, and thesis Charchov [6]).

- Berezin kernels are mostly studied in the context of Hermitian symmetric spaces. See e.g. [8], [30], [35], [37], [38], [39], [42], [45], [48], [52], [53].

The second model coming from the principal series representations of $S L(2, \mathbb{C})$ is wellsuited for generalizing canonical representations to para-Hermitian symmetric spaces (cf. $[33])$.

The most general definition of canonical representation is given in S. C. Hille's thesis [29]. It is built around arbitrary so-called $\sigma \theta$-invariant parabolic subgroups.

14. Interpolation. We return to the question posed in Section 9 about the interpolation of $\tau_{\lambda}$ between the $L^{2}$-spaces of $\mathrm{SU}(2) / K$ and $\mathrm{SU}(1,1) / K$. Notice that $\mathrm{SU}(1,1) / K$ can be viewed as an open submanifold of $\mathrm{SU}(2) / K \simeq \widetilde{S}$, see Section 12. This is in our view the main reason that we can relate both spaces in question. Let us now explain Neretin's solution in our own words and add some remarks. 
Let $\lambda$ be a negative integer. Then

$$
\psi_{\lambda}=\sum_{j=0}^{-\lambda} R_{j}(\lambda) \varphi_{1-2 \lambda-2 j}
$$

according to Section 9 .

Taking both sides at the point $g=a_{t}=\left(\begin{array}{c}\cosh t \sinh t \\ \sinh t \cosh t\end{array}\right)$, we get

$$
(\cosh t)^{-2 \lambda}=\sum_{j=0}^{-\lambda} R_{j}(\lambda) \varphi_{1-2 \lambda-2 j}\left(a_{t}\right) .
$$

One has

$$
\varphi_{s}\left(a_{t}\right)={ }_{2} F_{1}\left(\frac{s+1}{2}, \frac{-s+1}{2} ; 1 ;-\sinh ^{2} t\right) .
$$

For $s=1-2 \lambda-2 j$, being a positive odd integer, $\varphi_{s}\left(a_{t}\right)$ is a polynomial in $\cosh ^{2} t$, hence (14.1) also holds if we replace $\cosh t$ by $\cos t$. We get

$$
\psi_{\lambda}^{S U(2)}=\sum_{j=0}^{-\lambda} R_{j}(\lambda) \varphi_{1-2 \lambda-2 j}^{S U(2)}
$$

where

$$
\psi_{\lambda}^{S U(2)}(u)=\left|\left(u e_{1}, e_{1}\right)\right|^{-2 \lambda} \quad(u \in \mathrm{SU}(2))
$$

and $\varphi_{1-2 \lambda-2 j}^{S U(2)}$ is defined on $\mathrm{SU}(2)$ by setting its radial part equal to

$$
{ }_{2} F_{1}\left(1-\lambda-j, \lambda+j ; 1 ; \sin ^{2} t\right) \text {. }
$$

Observe that $\varphi_{1-2 \lambda-2 j}^{S U(2)}$ is a spherical function on $\mathrm{SU}(2)$.

Set $v=1-2 \lambda-2 j$. Then (14.2) can be rewritten as

$$
\psi_{\lambda}^{S U(2)}=\sum_{\substack{v=1 \\ v \text { odd }}}^{-2 \lambda+1} R_{\frac{1-2 \lambda-v}{2}}(\lambda) \varphi_{v}^{S U(2)} .
$$

Notice that

$$
R_{\frac{1-2 \lambda-v}{2}}(\lambda)=\frac{v \Gamma(1-\lambda)^{2}}{\Gamma\left(1-\lambda+\frac{1-v}{2}\right) \Gamma\left(1-\lambda+\frac{1+v}{2}\right)} .
$$

Call this expression $r_{v}(\lambda)$. One easily computes

$$
\int_{\mathrm{SU}(2)} \psi_{\lambda}^{S U(2)}(u) d u=\frac{1}{1-\lambda} .
$$

Let us now compute the asymptotic behaviour of $(1-\lambda) \psi_{\lambda}$ for $\lambda \rightarrow-\infty$. This comes down to determining the asymptotics of $(1-\lambda) r_{v}(\lambda)$ for fixed $v(v=1,3,5, \ldots)$.

Applying formula (8.1) we obtain:

$$
(1-\lambda) r_{v}(\lambda)=v\left\{1+\frac{v^{2}-1}{4 \lambda}+\mathcal{O}\left(\frac{1}{\lambda^{2}}\right)\right\} \quad(\lambda \rightarrow-\infty, \lambda \text { integer }) .
$$

Observe that $v$ runs over the set of dimensions of the finite-dimensional spherical representations of $\mathrm{SU}(2)$. Clearly (14.3) is equivalent to the following identity of distributions 
on $\widetilde{S}$ :

$$
(1-\lambda) \psi_{\lambda}^{S U(2)}=\delta+\frac{\square \delta}{4 \lambda}+\mathcal{O}\left(\frac{1}{\lambda^{2}}\right) \quad(\lambda \rightarrow-\infty, \lambda \text { integer }) .
$$

where $\square$ is now the Laplace-Beltrami operator on $\widetilde{S}$.

REMARK. One has for $\lambda$ negative integer:

$$
\psi_{\lambda}^{S U(2)}=\sum_{\substack{v=1 \\ v \text { odd }}}^{\infty} r_{v}(\lambda) \varphi_{v}
$$

Indeed, notice that $r_{v}(\lambda)=0$ for $v$ odd, $v>-2 \lambda+1$. It is easily checked that (14.5) remains true for all complex $\lambda$ with $\operatorname{Re} \lambda<1 / 2$ in $L^{2}$-sense, by computing inner products and using formulae from [21]. Neretin applies Carlson's theorem (Theorem 9.1), which is more convenient. Clearly (14.3) and (14.4) now hold without the restriction " $\lambda$ integer".

A final observation is that $\psi_{\lambda}^{S U(2)}$, for $\lambda$ a negative integer, is a positive-definite function associated with the representation $V_{-\lambda} \otimes \bar{V}_{-\lambda}$, where $V_{-\lambda}$ is the standard representation of $\mathrm{SU}(2)$ on holomorphic (harmonic) polynomials in $z_{1}, z_{2}$, homogeneous of degree $-\lambda$.

\section{References}

[1] J. Arazy and B. Ørsted, Asymptotic expansions of Berezin forms, preprint, 1999.

[2] F. A. Berezin, Quantization, Math. USSR Izv. 8 (1974), 1109-1165.

[3] F. A. Berezin, Quantization in complex symmetric space, Math. USSR Izv. 9 (1975), 341-379.

[4] F. A. Berezin, Connection between co- and contravariant symbols of operators on the classical complex symmetric spaces, Dokl. Akad. Nauk SSSR 241 (1978), 15-17 (in Russian); English transl.: Soviet Math. Dokl. 19 (1978), 786-789.

[5] F. A. Berezin and F. I. Karpelevic, Zonal spherical functions and Laplace operators on some symmetric spaces, Dokl. Akad. Nauk SSSR (N.S.) 118 (1958), 9-12 (in Russian).

[6] I. Charchov, Harmonic analysis on line bundles over complex hyperbolic spaces, Thesis, Leiden University, 2000.

[7] G. van Dijk, Group representations on spaces of distributions, Russian J. Math. Phys. 2 (1993), 57-68.

[8] G. van Dijk, Berezin kernels on tube domains, Report W97-07, University of Leiden, 1997; extended version 1999 (with M. Pevzner), J. Funct. Anal. 181 (2001), 189-208.

[9] G. van Dijk, Canonical representations associated to hyperbolic spaces II, Indag. Math. N.S. 10 (3) (1999), 357-368.

[10] G. van Dijk and S. C. Hille, Canonical representations related to hyperbolic spaces, J. Funct. Anal. 147 (1997), 109-139.

[11] G. van Dijk and S. C. Hille, Maximal degenerate representations, Berezin kernels and canonical representations, in: Lie Groups and Lie Algebras, Their Representations, Generalizations and Applications. Kluwer, Dordrecht, 1997.

[12] G. van Dijk and V. F. Molchanov, Tensor products of maximal degenerate series representations of the group $\mathrm{SL}(n, \mathbf{R})$, J. Math. Pures Appl. 78 (1999), 99-119. 
[13] G. van Dijk and V. F. Molchanov, The Berezin form for rank one para-Hermitian symmetric spaces, J. Math. Pures Appl. 77 (1998), 747-799.

[14] G. van Dijk and A. A. Pasquale, Canonical representations of $\operatorname{Sp}(1, n)$ associated with representations of $\mathrm{Sp}(1)$, Comm. Math. Phys. 202 (1999), 651-667.

[15] M. Engliš, Functions invariant under the Berezin transform, J. Funct. Anal. 121 (1994), 223-254.

[16] M. Engliš, Asymptotics of the Berezin transform and quantization on planar domains, Duke Math. J. 79 (1995), 57-76.

[17] M. Engliš and J. Peetre, On the correspondence principle for the quantized annulus, Math. Scand. 78 (1996), 183-206.

[18] M. Engliš, Berezin quantization and reproducing kernels on complex domains, Trans. Amer. Math. Soc. 348 (1996), 411-479.

[19] M. Engliš, Invariant operators and the Berezin transform on Cartan domains, Math. Nachr. 195 (1998), 61-75.

[20] A. Erdelyi et al., Higher Transcendental Functions, Vol. I, McGraw-Hill, New York, 1953.

[21] A. Erdelyi et al., Tables of Integral Transforms, Vol. II, McGraw-Hill, New York, 1954.

[22] J. Faraut and A. Korányi, Function spaces and reproducing kernels on bounded symmetric domains, J. Funct. Anal. 88 (1990), 64-89.

[23] J. Faraut and A. Korányi, Analysis on Symmetric Cones, Oxford Univ. Press, 1994.

[24] J. Faraut and G. Olafsson, Causal semisimple symmetric spaces, the geometry and harmonic analysis, in: Hofmann, Lawson and Vinberg (eds.), Semigroups in Algebra, Geometry and Analysis, de Gruyter, Berlin, 1995.

[25] E. Fujita and T. Nomura, Spectral decompositions of Berezin transformations on $\mathbb{C}^{n}$ related to the natural $U(n)$-action, J. Math. Kyoto Univ. 36 (1996), 877-888.

[26] E. Fujita and T. Nomura, Berezin transforms on the $2 \times 2$ matrix space related to the $U(2) \times U(2)$-action, Integral Eq. Oper. Theory 32 (1998), 152-179.

[27] L. I. Grosheva, Representations on distributions on the Lobachevsky plane concentrated at the boundary, Tambov University Reports 3(1) (1998), 46-49.

[28] S. C. Hille, Decomposition of tensor products of scalar unitary holomorphic and antiholomorphic representations of the universal covering group of $\mathrm{SU}(1, n)$, Report W98-12, University of Leiden, 1998.

[29] S. C. Hille, thesis, Leiden University, 1999.

[30] H. Ishi and T. Nomura, Spherical Fourier transforms of the Berezin kernels on symmetric Siegel domains, preprint 2000.

[31] P. E. T. Jorgensen and G. Olafsson, Unitary representations of Lie groups with reflection symmetry, J. Funct. Anal. 158 (1998), 26-88.

[32] P. E. T. Jorgensen and G. Olafsson, Unitary representations and Osterwalder-Schrader duality, in: The Mathematical Legacy of Harish-Chandra. A Celebration of Representation Theory and Harmonic Analysis. PSPM 68, 2000.

[33] V.F. Molchanov, Quantization on para-Hermitian symmetric spaces, in: Adv. in Math. Sci., AMS Transl. 175 (1996), 81-96.

[34] Yu. A. Neretin and G. I. Ol'shanskii, Boundary values of holomorphic functions, singular unitary representations of the group $O(p, q)$ and their limits as $q \rightarrow \infty$, Zap. Nauchn. Semin. POMI RAN 233 (1995), 9-91 (in Russian); English transl.: J. Math. Sci. 1996.

[35] Yu. A. Neretin, Boundary values of holomorphic functions and some special problems for unitary representations, in: J. Hilgert, J. D. Lawson, K.-H. Neeb and E. B. Vinberg (eds.), 
Positivity in Lie Theory: Open Problems, De Gruyter Expos. Math. 26, de Gruyter, Berlin, 1998, 221-248.

[36] Yu. A. Neretin, Pseudoriemannian symmetric spaces: one-type realizations and open embeddings to grassmannians, preprint, 1999.

[37] Yu. A. Neretin, Matrix analogs of the integral $\int_{0}^{\infty} \frac{x^{\alpha-1} d x}{(1+x)^{\rho}}=B(\alpha, \rho-\alpha)$ and Plancherel formula for Berezin kernel representations, preprint, 1999.

[38] T. Nomura, Berezin transforms and group representations, J. Lie Theory 8 (1998), 433440.

[39] T. Nomura, Invariant Berezin transforms, preprint, Kyoto University, 1999.

[40] T. Nomura, Berezin transforms and Laplace-Beltrami operators on homogeneous Siegel domains, preprint, 2000.

[41] G. Olafsson and B. Ørsted, Generalizations of the Bargmann transform, preprint, 1999.

[42] G. Olafsson, Analytic continuation in representation theory and harmonic analysis, preprint, 2000.

[43] B. Ørsted and G. Zhang, Weyl quantization and tensor products of Fock and Bergman spaces, Indiana Univ. Math. J. 43 (1994), 551-583.

[44] B. Ørsted and G. Zhang, $L^{2}$-versions of the Howe correspondence I, Math. Scand. 80 (1997), 115-160.

[45] B. Ørsted and G. Zhang, Tensor products of analytic continuations of holomorphic discrete series, Canad. J. Math. 49 (1997), 1224-1241.

[46] J. Peetre, The Berezin transform and Ha-plitz operators, J. Operator Theory 24 (1990), $165-186$.

[47] J. Repka, Tensor products of holomorphic discrete series representations, Canad. J. Math. 31 (1979), 836-844.

[48] A. Unterberger and H. Upmeier, Berezin transform and invariant differential operators, Comm. Math. Phys. 164 (1994), 563-598.

[49] H. Upmeier, Jordan Algebras in Analysis, Operator Theory, and Quantum Mechanics, CBMS-AMS Reg. Conf. Ser. in Math. 67, 1987.

[50] A. M. Vershik, I. M. Gel'fand and M. I. Graev, Representations of the group SL(2, R) where $\mathbf{R}$ is a ring of functions, Uspekhi Mat. Nauk 28 (5) (1973), 83-128 (in Russian); English transl.: Russian Math. Surv. 28 (5) (1973), 87-132.

[51] A. M. Vershik, I. M. Gel'fand and M. I. Graev, Representations of the group SL $(2, \mathbf{R})$ where $\mathbf{R}$ is a ring of functions, in: Representation Theory, London Math. Soc. Lecture Note Ser. 69, Cambridge Univ. Press, 1982.

[52] G. Zhang, Berezin transform on line bundles over bounded symmetric domains, preprint, 1998.

[53] G. Zhang, Tensor products of minimal holomorphic representations, preprint, 2000. 